\title{
Partial FFT Demodulation: A Detection Method for Highly Doppler Distorted OFDM Systems
}

\author{
Srinivas Yerramalli, Student Member, IEEE, Milica Stojanovic, Fellow, IEEE, and Urbashi Mitra, Fellow, IEEE
}

\begin{abstract}
Employing Orthogonal Frequency Division Multiplexing (OFDM) signaling over time-varying channels results in inter-carrier interference (ICI) and degraded detection error probability due to the loss of orthogonality among the subcarriers. This problem is particularly exacerbated for systems operating in highly mobile scenarios such as underwater acoustic (UWA) communications, digital video broadcasting (DVB) for mobile devices and vehicle-to-vehicle (V2V) networks. To address the problem of data detection in such scenarios, we propose a novel demodulation strategy using several partial interval Fast Fourier Transforms (FFTs) instead of a conventional, single full interval FFT. Algorithms for computing the weights used to combine the outputs of the partial FFT are presented for three scenarios: full, partial and no knowledge of the time varying channel. Numerical simulations and an approximate theoretical analysis show that significant performance gains can be obtained over traditional equalizers at a very moderate complexity.
\end{abstract}

Index Terms-Doppler compensation, OFDM signaling, partial FFT, time-varying channels, underwater acoustic communications.

\section{INTRODUCTION}

$\mathbf{O}$ RTHOGONAL Frequency Division Multiplexing (OFDM) is now the primary signaling scheme for several wireless communication systems such as Long Term Evolution (LTE), WiMAX, Digital Video Broadcasting (DVB) etc [4]-[6] and is also under consideration for underwater acoustic (UWA) communications [1], [7]-[9]. Interest in OFDM stems from the fact that it decomposes a static frequency selective channel into a number of flat channels, enabling low complexity, single-tap equalization and symbol-by-symbol detection at the receiver. A considerable amount of research on OFDM receivers for highly time-varying scenarios has been conducted. Such channels typically result when either there is high mobility or in wideband

Manuscript received October 17, 2011; revised February 26, 2012 and June 04, 2012; accepted June 29, 2012. Date of publication July 27, 2012; date of current version October 09, 2012. The associate editor coordinating the review of this manuscript and approving it for publication was Prof. Martin Haardt. This work was supported in part by ONR N00014-10-1-0576, ONR N00014-07-10738, ONR N00014-09-1-0700. Parts of this work have been presented at the International Workshop on Signal Processing and Advances in Wireless Communications (SPAWC) 2010, the Fourth ACM International Workshop on UnderWater Networks (WUWNet '10) 2010, and the IEEE Asilomar Conference on Signals, Systems, and Computers, 2010.

S. Yerramalli and U. Mitra are with the Department of Electrical Engineering, University of Southern California, Los Angeles, CA 90007 USA (e-mail: srinivas.yerramalli@usc.edu; ubli@usc.edu).

M. Stojanovic is with the Department of Electrical and Computer Engineering, Northeastern University, Boston, MA 02115 USA (e-mail: millitsa@ece.neu.edu).

Color versions of one or more of the figures in this paper are available online at http://ieeexplore.ieee.org.

Digital Object Identifier 10.1109/TSP.2012.2210547 signaling such as UWA communications where the transmission bandwidth is large relative to the carrier frequency. The Doppler in such highly mobile environments destroys the orthogonality of subcarriers, that results in inter-carrier interference (ICI), and significantly increases detection complexity required to mitigate the induced interference.

The problem of low complexity detection of OFDM signals over time-varying channels has been extensively studied in the literature. Conventional minimum mean-squared error (MMSE) based block equalizers have a complexity that grows cubically in block length and hence challenge practical implementation [10]. By exploiting the banded nature of the frequency domain channel matrix, several detection techniques whose complexity grows linearly in the number of sub-carriers have been designed. The performance of these receivers has been further enhanced through iterative detection and interference cancelation algorithms (see [9]-[15] and references therein for a detailed overview of time-varying channel equalization for OFDM). In general, lower complexity channel estimation and data detection algorithms are the key to implementing next generation OFDM systems with a large number of subcarriers.

In addition to the terrestrial wireless systems noted above, UWA communications also experience highly time-varying channels. The low speed of sound in water $(1500 \mathrm{~m} / \mathrm{s})$ coupled with mobility results in Doppler distortion, i.e., time scaling of the transmitted signal [8], [9], [13], [16]. The Doppler scaling in UWA communications is similar to that of OFDM radar systems tracking a single target [17]. The time scaling of the signal causes different subcarriers to be shifted by slightly different frequencies, resulting in significant ICI. Distortion compensation and equalization of OFDM signals for UWA channels have been extensively investigated in [2], [8], [9], [16], [18], [19].

In this paper, we revisit the problem of data detection over highly time-varying channels and propose a new demodulation technique called partial FFT demodulation. The received OFDM symbol is first partitioned into several intervals using non-overlapping rectangular windows and a discrete Fourier transform (DFT) ${ }^{1}$ is performed on each windowed segment of the received signal. The segments are then weighted and combined. If no weighting is applied, i.e., if the partial FFT outputs for each segment are directly added, the result is equivalent to performing conventional, full FFT demodulation, which results in significant ICI due to uncompensated Doppler distortion. In contrast, by judicious weighted combining of the partial FFT outputs, we show that the ICI can be significantly reduced, improving the detection performance at a complexity that is com-

\footnotetext{
${ }^{1}$ The DFT is efficiently implemented using the fast Fourier transform (FFT) and the DFT operation will be referred to as the FFT in the rest of the paper.
} 
parable to that of typical ICI equalization. The key to improved performance is that the partial FFT outputs contain less mixing of contributions from different symbols and allow for more effective compensation of ICI versus compensating after matched filtering. It should be noted that while we focus on the interference mitigation using partial FFT processing, further equalization is possible as well. However, for many practical applications, the benefits of improved front-end processing are sufficiently large that they obviate the need for subsequent equalization.

In [20], a windowing technique similar to partial FFT technique has been proposed which post-processes and equalizes the received signal by approximating the frequency domain spectrum of a rectangular window. In comparison, our work approximates the time-variation of the channel using a time-domain windowed version of the received signal and develops equalization techniques well suited for UWA communications. While [20] does not analyze the proposed scheme, this paper presents a detailed theoretical analysis for the proposed equalizers. In [21], an orthogonal chirp type signal basis is used to approximate the ideal signal basis functions. The chirp type functions correspond to the signal basis of the fractional Fourier transform (FRFT) in comparison to the sinusoidal basis of the regular Fourier transform. In effect, a multicarrier system similar to OFDM is designed using the FRFT instead of a conventional FFT. A generalization of [21], using the affine Fourier transform as an alternative to the FRFT was proposed in [22] and was shown to have more desirable properties than FRFT based multicarrier systems. Several researchers (see [23] and references therein) have also explored the design of orthogonal pulse shapes using a short-time Fourier (STF) basis for multicarrier communications. We distinguish our work from [21]-[23] by pointing out that we neither design new pulse shapes nor perform alternative transforms to the FFT at the receiver. The proposed method in this paper is a technique which exploits the structure in the time-variation and pre-processes the input signal by exploiting FFT processing to reduce time-variation.

We focus primarily on two scenarios in this paper: (1) UWA channels with time scaling distortion and (2) fast varying terrestrial radio channels experienced by users moving at a very high speed. We first illustrate the partial FFT combining technique and then derive the optimal weighting coefficients for combining the outputs for a generic model of the time-varying channel. The optimal combiner weights are shown to depend only on the channel frequency response at the midpoint of each interval. When the knowledge of the channel impulse response is not available at the receiver, we present a recursive weight estimation algorithm to compute the combiner weights. In UWA channels where the effect of Doppler distortion (time scaling) can be parameterized, the signal structure can be exploited to derive a model-based weight estimation algorithm for the combiner. We present an approximate analysis of the recursive and the model based estimators to determine the optimal number of partial FFTs to be employed in practice. Numerical simulations are presented to illustrate the performance of the proposed algorithms. We show that for signaling over the UWA channel with time scale distortion, the partial FFT method significantly outperforms banded MMSE equalizers and gives comparable performance to algorithms such as MCMC based MAP-SDSC in
[15] at a much lower complexity. For scenarios with no channel knowledge or with just knowledge of the distortion process, we show partial FFT processing performs significantly better than algorithms utilizing similar knowledge of the channel at a lower complexity. Finally, for the DVB scenario we show that the proposed method outperforms the conventional banded equalizers in most regimes of interest.

The paper is organized as follows. Section II presents the OFDM signal model and illustrates the concept of partial FFT demodulation. The optimal combiner weights, and the recursive weight estimation algorithm for scenarios with no prior channel knowledge are derived in Section III, while Section IV illustrates the model-based weight estimator for the UWA channel with Doppler distortion. Section V presents an analysis of the recursive and model-based estimation algorithms, and Section VI shows numerical results that demonstrate the effectiveness of this method in various scenarios. The paper concludes with remarks in Section VII.

\section{Signal Model and Partial FFT Demodulation}

\section{A. Signal Model}

Let us consider an OFDM system with $K$ subcarriers. The vector $\mathbf{d}=\left[d_{1}, d_{2}, \ldots, d_{K}\right]$ of information symbols is modulated onto the $K$ OFDM subcarriers. The transmitted symbols are assumed to be drawn from a finite constellation, such as 4-PSK, which we consider for illustration in this paper. Let $T$, $T_{g}$ and $\Delta f=\frac{1}{T}$ denote the duration of the OFDM symbol, duration of the cyclic prefix, and subcarrier spacing respectively. The $k^{t h}$ subcarrier frequency is $f_{k}=f_{0}+(k-1) \Delta f$, and the total OFDM signaling bandwidth is $B=K \Delta f$. The transmitted OFDM signal in passband can be expressed as

$$
s(t)=\sqrt{\frac{1}{T}} \operatorname{Re}\left\{\sum_{l=1}^{K} d_{l} e^{j 2 \pi f_{l} t}\right\}, t \in\left[-T_{g}, T\right] .
$$

The passband time-varying channel is modeled as

$$
h(\tau, t)=\sum_{p=1}^{P} h_{p}(t) \delta\left(\tau-\tau_{p}(t)\right),
$$

where $h_{p}(t)$ and $\tau_{p}(t)$ are the time-varying gain and delay of the $p^{\text {th }}$ path, respectively, and $P$ is the total number of arriving paths. The received signal in passband can then be expressed as

$$
\begin{aligned}
r(t)=\sqrt{\frac{1}{T}} \operatorname{Re}\left\{\sum_{p=1}^{P} \sum_{l=1}^{K} h_{p}(t) d_{l} e^{j 2 \pi f_{l}\left(t-\tau_{p}(t)\right)}\right\} \\
+z(t), t \in\left[-T_{g}, T\right]
\end{aligned}
$$

where $z(t)$ is additive white Gaussian noise (AWGN). 


\section{B. Conventional OFDM Demodulation}

In a conventional receiver, after time and frequency synchronization, the cyclic prefix is discarded and the received signal is transformed into the frequency domain: ${ }^{2}$

$$
\begin{aligned}
r_{k}= & \frac{1}{\sqrt{T}} \int_{0}^{T} r(t) e^{-j 2 \pi f_{k} t} d t \\
= & \frac{1}{T} \sum_{l=1}^{K} d_{l} \int_{0}^{T} \underbrace{\left[\sum_{p=1}^{P} h_{p}(t) e^{-j 2 \pi f_{l} \tau_{p}(t)}\right]}_{H_{l}(t)} \\
& \times e^{j 2 \pi\left(f_{l}-f_{k}\right) t} d t+z_{k} .
\end{aligned}
$$

Here, $H_{l}(t)$ represents the time-varying frequency response of the $l^{t h}$ subcarrier, and $z_{k}$ is the AWGN with zero mean and variance $N_{0}$. From (4), we clearly observe how the time-varying channel causes ICI. When $H_{l}(t)$ is time-invariant, the received signal on each subcarrier reduces to $r_{k}=d_{k} H_{k}+z_{k}$, enabling one-tap equalization and making symbol-by-symbol detection optimal. The time variation in $H_{l}(t)$ destroys this orthogonality, and necessitates ICI equalization to compensate for the timevarying channel [10], [11].

\section{Partial FFT Demodulation}

In partial FFT demodulation, the useful OFDM symbol duration $[0, T]$ is divided into $M$ non-overlapping intervals (equivalent to multiplying the signal with several rectangular windows) and a Fourier transform is performed on each windowed segment of the signal. The output of the Fourier transform for the $k^{t h}$ subcarrier and the $m^{t h}$ windowed block, henceforth called the partial FFT output, can be expressed as

$$
\begin{aligned}
y_{k}(m) & =\sqrt{\frac{1}{T}} \int_{\frac{(m-1) T}{M}}^{\frac{m T}{M}} r(t) e^{-j 2 \pi f_{k} t} d t \\
& =\frac{1}{T} \sum_{l} d_{l} \int_{\frac{(m-1) T}{M}}^{\frac{m T}{M}} H_{l}(t) e^{j 2 \pi\left(f_{l}-f_{k}\right) t} d t+z_{k}(m), \\
m & =1,2, \ldots, M .
\end{aligned}
$$

In general, it is assumed that the channel parameters vary slowly in comparison to the OFDM symbol duration. We exploit this assumption by approximating the time-varying frequency response in each interval $\frac{T}{M}$ by the midpoint value of the function. The received signal (5) can now be simplified as

$$
\begin{aligned}
& y_{k}(m) \approx \sum_{l=1}^{K} d_{l} H_{l}(m) I_{l-k}(m)+z_{k}(m), \\
& H_{l}(m)=\sum_{p=1}^{P} h_{p}(m) e^{-j 2 \pi f_{l} \tau_{p}(m)} .
\end{aligned}
$$

where $H_{l}(m), h_{p}(m)$ and $\tau_{p}(m)$ are the midpoint values of the frequency response, the channel impulse response and path delays, respectively, on the interval $\left[(m-1) \frac{T}{M}, \frac{m T}{M}\right]$. The func-

\footnotetext{
${ }^{2}$ We use the continuous time Fourier transform for simplicity. In practice as well as in our numerical simulations, FFT is used.
}

tion $I_{i}(m)$ captures the effect of partial integration over the $m^{t h}$ interval, and can be evaluated as

$$
\begin{aligned}
I_{i}(m) & =\frac{1}{T} \int_{\frac{(m-1) T}{M}}^{\frac{m T}{M}} e^{j 2 \pi i \Delta f t} d t \\
& =\frac{1}{M} e^{\frac{j 2 \pi i(2 m-1)}{(2 M)}} \operatorname{sinc}\left(\frac{\pi i}{M}\right), \\
i & =-(K-1), \ldots(K-1),
\end{aligned}
$$

where $\operatorname{sinc}(x) \doteq \frac{\sin (x)}{x}$. This function has the property that $I_{0}(m)=\frac{1}{M}, m=1,2, \ldots, M$ and $\sum_{m=1}^{M} I_{i}(m)=0 \forall i \neq 0$ We will exploit this property later when we design the combiner.

The noise is characterized by the covariances

$$
\begin{aligned}
& E\left[z_{k_{1}}\left(m_{1}\right) z_{k_{2}}^{*}\left(m_{2}\right)\right] \\
& = \begin{cases}\frac{N_{0}}{M} e^{-\frac{j \pi\left(k_{1}-k_{2}\right)(2 m-1)}{M}} \operatorname{sinc}\left(\frac{\pi\left(k_{1}-k_{2}\right)}{2 M}\right) & \text { if } m_{1}=m_{2}=m \\
0 & m_{1} \neq m_{2} .\end{cases}
\end{aligned}
$$

The noise components are thus Gaussian and uncorrelated and hence independent across partial FFT outputs for a given subcarrier, but are correlated for a fixed $m$ across subcarriers. For the $k^{t h}$ subcarrier, let us now define $\mathbf{v}_{k}=\left[I_{k}(1), I_{k}(2), \ldots, I_{k}(M)\right]^{T}$ as the vector containing the partial interval integration coefficients, $\mathbf{H}_{k}=\operatorname{diag}\left[H_{k}(1), H_{k}(2), \ldots, H_{k}(M)\right]$ as the diagonal matrix containing the channel frequency response in each interval, and $\mathbf{y}_{k}=\left[y_{k}(1), y_{k}(2), \ldots, y_{k}(M)\right]^{T}$ as the vector of partial FFT outputs. ${ }^{3}$ Expressing (6) in vector form, we have

$$
\mathbf{y}_{k}=\sum_{l=1}^{K} d_{l} \mathbf{H}_{l} \mathbf{v}_{l-k}+\mathbf{z}_{k}
$$

Note that $\mathbf{v}_{0}=\frac{1}{M}[1,1, \ldots, 1]^{T}$ and $\mathbf{v}_{k}^{H} \mathbf{v}_{0}=0 \forall k \neq 0$ which compactly expresses the fact that the OFDM subcarriers are orthogonal to each other.

\section{Combining Partial FFT Outputs}

Let us define $\mathbf{w}_{k}=\left[w_{k}(1), w_{k}(2), \ldots, w_{k}(M)\right]^{T}$ as the vector of the combiner weights for the $k^{\text {th }}$ subcarrier. The combining then yields

$$
x_{k}=\mathbf{w}_{k}^{H} \mathbf{y}_{k}=\sum_{l=1}^{K} d_{l} \mathbf{w}_{k}^{H} \mathbf{H}_{l} \mathbf{v}_{l-k}+\mathbf{w}_{k}^{H} \mathbf{z}_{k} .
$$

An appropriate choice of the vectors $\mathbf{w}_{k}$ allows one to compensate for the time-variation of the channel to some degree, thus reducing the ICI but not completely eliminating it. Even if one were to implement optimal front-end filtering, (i.e., a matched filter for each subcarrier), the resulting output would contain ICI. Partial FFT demodulation followed by optimized combining mimics the operation of optimal front-end filtering and cannot completely eliminate the ICI. However, it can significantly reduce its effect possibly even eliminating the need

\footnotetext{
${ }^{3}$ Notation: $\mathbf{a}^{T}$ denotes the transpose of $\mathbf{a}$, and $\mathbf{a}^{H}$ denotes its conjugate trans-
} pose. 
for post-FFT ICI equalization. Clearly, partial FFT demodulation can also be combined with ICI equalization; however, our goal in this paper is to investigate its performance when used as a stand-alone alternative to equalization of full FFT outputs.

\section{E. Partial FFT Combining: A Window Design Interpretation}

The proposed technique can be alternatively interpreted by recasting the problem as designing a receiver window for each subcarrier. For ease of illustration, we briefly consider a discrete time equivalent model of the received signal. Let us suppose that $r(k)$ is the sampled version of the signal $r(t)$ and $\mathbf{r}=[r(1), r(2), \ldots, r(K)]$. The partial FFT outputs can be expressed in terms of $\mathbf{r}$ as

$$
y_{k}(m)=\mathbf{e}_{k}^{T} \mathbf{F} \operatorname{diag}\left\{\beta_{m}\right\} \mathbf{r},
$$

where $\mathbf{e}_{k}$ is a unit vector whose $k^{t h}$ entry is $1, \beta_{m}$ is the rectangular window used for computing the $m^{\text {th }}$ partial FFT output, and $\mathbf{F}$ is the $K \times K$ DFT matrix. Now, the combined partial FFT outputs in (10) can be written as

$$
\begin{aligned}
x_{k} & =\mathbf{w}_{k}^{H} \mathbf{y}_{k}=\sum_{m=1}^{M} w_{k}^{*}(m) y_{k}(m) \\
& =\mathbf{e}_{k}^{T} \mathbf{F} \underbrace{\left[\sum_{m=1}^{M} w_{k}^{*}(m) \operatorname{diag}\left\{\beta_{m}\right\}\right]}_{\overline{\mathbf{w}}_{k}} \mathbf{r}=e_{k}^{T} \mathbf{F}\left[\overline{\mathbf{w}}_{k}\right] \mathbf{r} .
\end{aligned}
$$

Clearly, each subcarrier has its own window, in contrast to the approach in [10], [12], where one window is used for all the subcarriers. In addition, the window $\overline{\mathbf{w}}_{k}$ is step-wise, while the windows in [10], [12] are in general smooth. Thus, the partial FFT technique can be considered as a generalization of the windowing method adopted in OFDM systems. Though [20] also proposes a similar windowing technique, it significantly differs from this work in the way the combiner coefficients $w_{k}(m)$ are determined.

\section{COMPuting the COMBINER Weights}

\section{A. Perfect Channel Knowledge Scenario}

To derive the MMSE minimizing combiner weights $\mathbf{w}_{k}^{\text {opt }}$, we consider the partial FFT outputs for the $k^{\text {th }}$ subcarrier to determine the symbol transmitted on this subcarrier. We emphasize that the only the $k^{\text {th }}$ partial FFT outputs are considered for computing $\mathbf{w}_{k}^{\text {opt }}$. Assuming that all the channel parameters are deterministic and known, the MMSE combiner weights are the solution to the optimization problem:

$$
\begin{aligned}
\mathbf{w}_{k}^{o p t} & =\min _{\mathbf{w}_{k}} E\left\{\left|d_{k}-\mathbf{w}_{k}^{H} \mathbf{y}_{k}\right|^{2}\right\} \Rightarrow \mathbf{w}_{k}^{o p t} \\
& =\left(E\left\{\mathbf{y}_{k} \mathbf{y}_{k}^{H}\right\}\right)^{-1} E\left\{\mathbf{y}_{k} d_{k}^{*}\right\} .
\end{aligned}
$$

Substituting for $\mathbf{y}_{k}$ from (9) and evaluating the cross-correlation and autocorrelation matrices, we obtain

$$
\begin{aligned}
\mathbf{R}_{\mathbf{y}_{k} d_{k}} & =E\left\{\mathbf{y}_{k} d_{k}^{*}\right\}=\mathbf{H}_{k} \mathbf{v}_{0}, \\
\mathbf{R}_{\mathbf{y}_{k}} & =E\left\{\mathbf{y}_{k} \mathbf{y}_{k}^{H}\right\} \\
& =\sum_{l=1}^{K} \mathbf{H}_{l} \mathbf{v}_{l-k} \mathbf{v}_{l-k}^{H} \mathbf{H}_{l}^{H}+\frac{N_{0}}{M} \mathbf{I}_{M} .
\end{aligned}
$$

The MMSE optimal combiner weights for the $k^{\text {th }}$ subcarrier can then be evaluated as $\mathbf{w}_{k}^{\text {opt }}=\mathbf{R}_{\mathbf{y}_{k}}^{-1} \mathbf{H}_{k} \mathbf{v}_{0}$.

1) Example-Time Invariant Channel: To gain some intuition, let us evaluate the combiner weights for the simple scenario of a linear time-invariant channel. The subcarrier frequency response coefficients $H_{l}(m)$ given by (6) are then equal in all the $M$ intervals. The elements of the auto-correlation matrix of the outputs of the $k^{t h}$ subcarrier are then

$$
\begin{aligned}
\mathbf{R}_{\mathbf{y}_{k}}\left(m_{1}, m_{2}\right)= & \frac{1}{M^{2}} \sum_{l=1}^{K}\left|H_{l}\right|^{2} e^{j 2 \pi(l-k) \frac{m_{1}-m_{2}}{M}} \\
& \times \operatorname{sinc}^{2}\left(\frac{\pi(l-k)}{M}\right)+\frac{N_{0}}{M} \delta\left(m_{1}-m_{2}\right) .
\end{aligned}
$$

Defining $\oplus$ as addition modulo $M$, we see that $\mathbf{R}_{\mathbf{y}_{k}}$ is a circulant matrix as $\mathbf{R}_{\mathbf{y}_{k}}\left(m_{1} \oplus M, m_{2} \oplus M\right)=\mathbf{R}_{\mathbf{y}_{k}}\left(m_{1}, m_{2}\right)$. Exploiting the fact that any circulant matrix can be diagonalized by a discrete Fourier transform (DFT) matrix, we get $\mathbf{R}_{\mathbf{y}_{k}}=\mathbf{F} \boldsymbol{\Lambda}_{k} \mathbf{F}^{H}$, where $\mathbf{F}$ is the $M \times M$ unitary DFT matrix and $\boldsymbol{\Lambda}_{k}$ is a diagonal matrix of the eigen-values of $\mathbf{R}_{\mathbf{y}_{k}}$. The optimal combiner coefficients now reduce to $\mathbf{w}_{k}^{o p t}=\frac{H_{k}}{M} \mathbf{F} \boldsymbol{\Lambda}_{k}^{-1} \mathbf{F}^{H} \mathbf{v}_{0}=\frac{H_{k}}{\boldsymbol{\lambda}_{k}(1)} \mathbf{v}_{0}$. The closed form solution is obtained by noting that the first column vector of the DFT matrix is $\mathbf{v}_{0}$ and $\boldsymbol{\lambda}_{k}(1)$ is the first diagonal element of $\boldsymbol{\Lambda}_{k}$. The eigenvalue $\boldsymbol{\lambda}_{k}(1)$, corresponding to the first eigenvector $\mathbf{v}_{0}$, can be evaluated from the first row of $\mathbf{R}_{\mathbf{y}_{k}}$ as $\lambda_{k}(1)=\sum_{i=1}^{M} \mathbf{R}_{\mathbf{y}_{k}}(1, i)=\frac{1}{M}\left(\left|H_{k}\right|^{2}+N_{0}\right)$ and the MMSE optimal combiner weights are

$$
\mathbf{w}_{k}^{o p t}=\frac{H_{k}}{\left|H_{k}\right|^{2}+N_{0}} M \mathbf{v}_{0}=\frac{H_{k}}{\left|H_{k}\right|^{2}+N_{0}}[1,1, \ldots, 1]^{T} .
$$

In other words, optimal processing for a time-invariant channel amounts to adding all the partial FFT outputs for a given subcarrier and then performing single-tap equalization - as expected, this is identical to conventional OFDM processing.

\section{B. No Channel Knowledge Scenario}

The MMSE estimate for the combiner weights in (13) relies on the knowledge of the channel frequency response at the midpoint of each of the $M$ partial intervals. Since estimating the time-varying channel impulse response may not always be practical, we propose a recursive weight (RW) estimation algorithm that does not utilize any knowledge of the time-varying channel.

We derive the estimator based on the assumption that the frequency response of the channel changes slowly across subcarriers, and thus the combiner weights $\mathbf{w}_{k}^{o p t}$ also change slowly as a function of the subcarrier index. This assumption is valid for OFDM systems whose coherence bandwidth is much greater than the subcarrier spacing. For example, this assumption has been exploited in [18] to design low complexity detection algorithms for underwater MIMO-OFDM systems.

The RW estimation algorithm is based on the idea that partial FFT combining eliminates most of the ICI and that the resulting output after combining for $k^{t h}$ subcarrier can be modeled as

$$
x_{k} \approx H_{k} d_{k}+w_{k},
$$

where $H_{k}$ is the channel frequency response and $w_{k}$ contains the noise and residual ICI.

Using the estimated values of $\mathbf{w}_{k}^{H}$, the partial FFT outputs $\mathbf{y}_{k}^{H}$ are combined to yield $x_{k}=\mathbf{w}_{k}^{H} \mathbf{y}_{k}$. Using an estimate of 
the frequency response on the previous subcarrier, $\hat{H}_{k-1}$, the received signal is equalized to form a preliminary estimate of the data symbol, and make the corresponding decision:

$$
\hat{d}_{k}=\frac{x_{k}}{\hat{H}_{k-1}} ; \tilde{d}_{k}=\operatorname{dec}\left(\hat{d}_{k}\right) .
$$

Now, using this coarse estimate of the transmitted symbols, the channel frequency response for the subcarrier of interest is updated as

$$
\hat{H}_{k}=\alpha \hat{H}_{k-1}+(1-\alpha) \frac{x_{k}}{\tilde{d}_{k}} .
$$

Assuming correct symbol decisions, or using pilots when available, the error at the combiner output is evaluated as

$$
e_{k}=\hat{H}_{k} \tilde{d}_{k}-x_{k} .
$$

This error is used to drive an adaptive algorithm for the combiner weights, for example the recursive least squares (RLS) algorithm:

$$
\mathbf{w}_{k+1}=\mathbf{w}_{k}+\operatorname{RLS}\left[y_{k}, e_{k}\right] .
$$

The detection method is completely summarized in Algorithm 1. The parameter $\alpha \in[0,1]$ controls the update of the channel frequency response and is dependent on the coherence bandwidth of the channel. For channels with small delay spreads, the coherence bandwidth is larger and the frequency response changes slowly across subcarriers. However for channels with large delay spreads, the coherence bandwidth is smaller and the frequency gains on subcarriers change rapidly from one subcarrier to another and a smaller value of $\alpha$ is suited for such scenarios. The parameter $\lambda$ is the forgetting factor of the RLS algorithm and is chosen to balance between channel dynamics and estimation noise and is typically very close to one.

The algorithm is initialized by choosing the channel frequency response to be used for the estimating data on the first subcarrier to be $H_{0}=1$. The initializing weight vectors are chosen to be $[1,1, \ldots, 1]^{T}$ as equal gain combining of the partial FFT outputs is optimal when the channel is time-invariant. The parameter $\delta$ which determines the initial covariance matrix $G_{1}$ is chosen so that the all the variables are independent and have a large variance (i.e., $\delta$ is very small) to simulate the fact that no prior information is available about the statistics of the partial FFT outputs. The first $2 M$ subcarriers are assigned to be pilots to operate the algorithm in pilot-assisted mode which allows better estimation of the RLS error $e_{k}$ to train the system parameters. The algorithm then switches to decision directed model wherein the estimated data symbols are used for computing the error and further updating the weight vector. Note that the inclusion of pilot symbols in the first few subcarriers reduces the rate of data transmission. It is shown in Sections $\mathrm{V}$ and VI that a small value of $M$ is optimal and as $2 M \ll K$, the rate reduction is negligible.

Insertion of pilots beyond those necessary for initial RLS convergence is required for channels that exhibit spectral nulls. On such channels, a subcarrier experiencing a deep fade may cause a symbol error, which will then propagate across subcarriers unless corrected. To overcome the loss of detection performance caused by error propagation, pilot symbols are inserted periodically throughout the OFDM symbol and knowledge of the pilot
Algorithm 1: Recursive Weight Estimation

1:INITIALIZATION:

2:Weights: $\mathbf{w}_{1}=[1,1, \ldots, 1]_{M \times 1}^{T}$

3:Covariance matrix: $\delta \ll 1, \mathbf{G}_{1}=\delta^{-1} \mathbf{I}_{M \times M}$

4:Control parameters: $\lambda=0.99, \alpha=0.2$

5:Channel estimates: $\hat{H}_{0}=1$

6:for $k=1$ to $K$ do

7:COMPUTE SIGNALS:

$8: \mathbf{y}_{k}=\left[y_{1}(k), \ldots, y_{M}(k)\right]^{T}$

9: $x_{k}=\mathbf{w}_{k}^{H} \mathbf{y}_{k}$

$10: \hat{d}_{k}=\frac{x_{k}}{\hat{H}_{k-1}}$

11:DATA DETECTION/PILOTS:

12:if $k \in\left\{k_{1}, k_{2}, \ldots, k_{P}\right\}$ then

$13: \tilde{d}_{k}=d_{k}$

14:else

$15: \tilde{d}_{k}=\operatorname{dec}\left(\hat{d}_{k}\right), \operatorname{dec}(\cdot)$ maps the point to the nearest constellation symbol.

\section{6 :end if}

17:UPDATE THE CHANNEL:

18: $\hat{H}_{k}=\alpha \hat{H}_{k-1}+(1-\alpha) \frac{x_{k}}{d_{k}}$

19:UPDATE THE COMBINER (RLS ALGORITHM):

$20: e_{k}=\hat{H}_{k} \tilde{d}_{k}-x_{k}$

$21: \mathbf{g}_{k}=\frac{\mathbf{G}_{k} \mathbf{y}_{k}}{\lambda+\mathbf{y}_{k}^{H} \mathbf{G}_{k} \mathbf{y}_{k}}$

$22: \mathbf{w}_{k+1}=\mathbf{w}_{k}+e_{k}^{*} \mathbf{g}_{k}$

$23: \mathbf{G}_{k+1}=\frac{1}{\lambda}\left(\mathbf{G}_{k}-\mathbf{g}_{k} \mathbf{y}_{k}^{H} \mathbf{G}_{k}\right)$.

24:end for

25:RE-COMPUTE FREQUENCY RESPONSE:

$26: \mathbf{x}^{p}=\left[x_{k_{1}}, x_{k_{2}}, \ldots, x_{k_{P}}\right]^{T}$

$27: \mathbf{S}^{p}=\operatorname{diag}\left[d_{k_{1}}, d_{k_{2}}, \ldots, d_{k_{P}}\right]$

$28: \hat{\mathbf{h}}=\mathbf{F}^{\dagger}\left(\mathbf{S}^{p}\right)^{-1} \mathbf{x}^{p}, \mathbf{F}_{m, l}=e^{j 2 \pi k_{m} \frac{l}{K}}$.

29: $\check{H}_{k}=\sum_{l=1}^{L} \hat{h}_{l} e^{-j 2 \pi k \frac{l}{K}}$

30:DATA DETECTION:

31: $\tilde{d}_{k}=\operatorname{dec}\left(\frac{x_{k}}{\tilde{H}_{k}}\right)$

symbol is used in place of estimated data symbols to update the channel frequency response.

Once the partial FFT outputs are combined using the RLS to drive the weight estimation, the receiver uses the distortion corrected outputs $x_{k}$ and the knowledge of the pilot symbols to compute an estimate of the channel impulse response $\hat{\mathbf{h}}$ as illustrated in Algorithm 1. The channel frequency response, computed from $\hat{\mathbf{h}}$ as $\check{H}_{k}=\sum_{l=1}^{L} \hat{h}_{l} e^{\frac{-j 2 \pi k l}{K}}$, can now be used to perform single-tap equalization on $x_{k}$ to recover estimates of the transmitted data symbols.

\section{Model Based Combining}

Previously, we have computed the combiner weights when either complete or no knowledge of the channel was available at the receiver. In this section, we investigate scenarios where partial knowledge of the distortion process, such as a parametric 
representation, is available to the receiver. In general the combiner weights do not have any structure and each weight has to be estimated independently. However, in some scenarios of interest such as UWA channels, we can obtain a parametric representation of the combiner weights. This parametrization considerably reduces the number of parameters to be estimated and enables simpler equalization.

To illustrate this method, let us consider OFDM signaling over an UWA channel with Doppler distortion wherein the received signal on each path is a time scaled version of the transmitted signal. Assuming that the channel coefficients are constant for the duration of one OFDM symbol, the time variation of the signal is captured by the time-varying path delays $\tau_{p}(t)=\tau_{p}-a t$, where $a=\frac{v}{c}$ is the ratio of the relative transmitter/receiver velocity to the speed of sound (see [8], [13], [16] and the references therein for the UWA channel model). From (3), the received signal in such a scenario can be expressed as

$$
r(t)=\frac{1}{\sqrt{T}} \operatorname{Re}\left[\sum_{l=1}^{K} d_{l} H_{l} e^{j 2 \pi f_{l} t(1+a)}\right]+z(t) .
$$

We observe that for a time-scaled OFDM signal, each subcarrier at frequency $f_{k}$ is shifted by an offset $a f_{k}$. This model can also be considered as a generalization of the OFDM system with carrier frequency offset (CFO) [24] where all the subcarriers are shifted by the frequency $f_{d} \mathrm{~Hz}$. The partial FFT outputs for the time scale scenario can be expressed as

$$
\begin{aligned}
\mathbf{y}_{k} & =\sum_{l=1}^{K} d_{l} H_{l} \mathbf{D}_{\epsilon_{l}} \mathbf{v}_{l-k}+\mathbf{n}_{k}, \epsilon_{l}=a f_{l} T \\
\mathbf{D}_{\epsilon_{l}} & =\operatorname{diag}\left[e^{j 2 \pi \epsilon_{l} \frac{(2 m-1)}{2 M}}\right], m=1,2, \ldots, M .
\end{aligned}
$$

For OFDM with CFO, the partial FFT outputs are obtained by substituting $\epsilon_{l}=\epsilon=f_{d} T, \forall l$. Before illustrating the model based weight estimator, we first compute the optimal combiner weights for the two scenarios.

1) OFDM With Carrier Frequency Offset: Evaluating the correlation and covariance matrices needed for the weights in (13) using (28) with $\epsilon_{l}=f_{d} T, \forall l$, we obtain

$$
\begin{aligned}
\mathbf{R}_{\mathbf{y}_{k}} & =\mathbf{D}_{\epsilon}\left[\sum_{l=1}^{K}\left|H_{l}\right|^{2} \mathbf{v}_{l-k} \mathbf{v}_{l-k}^{H}+\frac{N_{0}}{M} \mathbf{I}_{M}\right] \mathbf{D}_{\epsilon}^{H}, \\
\mathbf{R}_{\mathbf{y}_{k} d_{k}} & =\mathbf{D}_{\epsilon} H_{k} \mathbf{v}_{0} .
\end{aligned}
$$

Noting that $\mathbf{D}_{\epsilon} \mathbf{D}_{\epsilon}^{H}=\mathbf{I}_{M}$ and simplifying the resulting expression similarly as in the case of a time-invariant channel in Section II, the optimal combiner weights are found to be

$$
\mathbf{w}_{k}^{o p t}=\mathbf{D}_{\epsilon} \frac{H_{k}}{\left|H_{k}\right|^{2}+N_{0}} M \mathbf{v}_{0} .
$$

The structure of the optimal combiner suggests that the receiver first compensates for the phase shift $e^{\frac{j 2 \pi(2 m-1)}{(2 M)}}$ at the $m^{t h}$ partial FFT output and then combines them with equal weights before channel equalization. Note that this method does not completely eliminate ICI as the compensated phase shift does not completely eliminate the CFO.
2) OFDM With Time Scaling Distortion: Evaluating the auto-correlation and the cross-correlation terms as in the previous case, we obtain

$$
\begin{aligned}
\mathbf{R}_{\mathbf{y}_{k}} & =\sum_{l=1}^{K}\left|H_{l}\right|^{2} \mathbf{D}_{\epsilon_{l}} \mathbf{v}_{l-k} \mathbf{v}_{l-k}^{H} \mathbf{D}_{\epsilon_{l}}^{H}+\frac{N_{0}}{M} \mathbf{I}_{M}, \\
\mathbf{R}_{\mathbf{y}_{k} d_{k}} & =\mathbf{D}_{\epsilon_{k}} H_{k} \mathbf{v}_{0} .
\end{aligned}
$$

Expanding the individual entries of the above matrix, we get

$$
\begin{aligned}
\mathbf{R}_{\mathbf{y}_{k}}\left(m_{1}, m_{2}\right)= & \frac{1}{M^{2}} \sum_{l=1}^{K}\left|H_{l}\right|^{2} e^{j 2 \pi\left(l-k+\epsilon_{l}\right) \frac{\left(m_{1}-m_{2}\right)}{M}} \\
& \times \operatorname{sinc}^{2}\left(\frac{\pi(l-k)}{M}\right)+\frac{N_{0}}{M} \delta\left(m_{1}-m_{2}\right) .
\end{aligned}
$$

We observe that the autocorrelation matrix is not a circulant matrix and a simple closed form solution for the optimal weights $\mathbf{w}_{k}^{o p t}$ may be intractable. However, $\mathbf{R}_{\mathbf{y}_{k}}$ is a Toeplitz Hermitian matrix for each $k$. Consequently, we define the matrix $\mathbf{C}_{\mathbf{y}_{k}}=$ $\operatorname{circ}\left[\mathbf{R}_{\mathbf{y}_{k}}\left(1, m_{2}\right)\right], m_{2}=1,2, \ldots, M$ as the related circulant matrix of $\mathbf{R}_{\mathbf{y}_{k}}$. In the limit of large values of $M$, assuming that the strong norms of the inverses of $\mathbf{R}_{\mathbf{y}_{k}}$ and $\mathbf{C}_{\mathbf{y}_{k}}$ are bounded, we can approximate the Toeplitz matrix by its equivalent circulant matrix [25], and the optimal combiner coefficients in such a scenario are approximately

$$
\mathbf{w}_{k}^{o p t} \approx \mathbf{D}_{\epsilon_{k}} \frac{H_{k}}{\lambda_{k}(1)} M \mathbf{v}_{0} \approx \mathbf{D}_{\epsilon_{k}} \frac{H_{k}}{\left|H_{k}\right|^{2}+N_{0}} M \mathbf{v}_{0} \cdot
$$

where $\lambda_{k}(1)=\sum_{i=1}^{M} \mathbf{C}_{\mathbf{y}_{k}}(1, i)=\sum_{i=1}^{M} \mathbf{R}_{\mathbf{y}_{k}}(1, i)$ is the first eigenvalue of $\mathbf{C}_{\mathbf{y}_{k}}$. Note that computing $\lambda_{k}(1)$ requires the knowledge of the channel frequency response for each subcarrier and the time scale parameter $a$. Clearly, the strategy of first compensating for the phase distortion and then equalizing the received signal is asymptotically optimal. Even though this strategy is not optimal, it will be later shown through simulations that the combiner weights computed using (28) result in significant ICI reduction even for small values of $M$.

We now propose an estimator which exploits the parametric model for the combiner weights in (28) to detect data transmitted using OFDM signals over a UWA channel. As the estimator relies on a model of the combiner weights, this estimator is called a model-based weight (MW) estimator.

\section{A. Model-Based Weight Estimation}

Let us define $\left\{k_{1}, k_{2}, \ldots, k_{P}\right\}$ as the set of subcarriers carrying pilot symbols. Using the model for the received signal in (23), the combiner weights are designed to compensate for the phase rotation due to the time scaling. Assuming $\tilde{a}$ to be a candidate value of the time scaling factor, let us define the diagonal matrix $\mathbf{D}_{\tilde{a}}(m)=\operatorname{diag}\left[e^{j 2 \pi f_{k_{1}} \tilde{a} \frac{2 m-1}{2 M} T}, \ldots, e^{j 2 \pi f_{k_{P}} \tilde{a} \frac{2 m-1}{2 M} T}\right]$ to model the phase rotation for the $m^{\text {th }}$ partial FFT interval. The partial FFT outputs for the pilot subcarriers for this candidate value of $\tilde{a}$ are combined as

$$
\mathbf{x}_{\tilde{a}}^{p}=\sum_{m=1}^{M} \mathbf{D}_{\tilde{a}}^{H}(m) \mathbf{y}^{p}(m),
$$


where the vector $\mathbf{y}^{p}(m)=\left[y_{k_{1}}^{p}(m), \ldots, y_{k_{P}}^{p}(m)\right]$ contains the elements of the partial FFT outputs for the pilot subcarriers in the $m^{t h}$ interval. Assuming that this candidate value is the correct value of the time scale factor and that $\mathbf{x}_{\tilde{a}}^{p}$ is nearly free of distortion, the combined outputs for the pilot subcarriers can be expressed as

$$
\mathbf{x}_{\tilde{a}}^{p}=\mathbf{S}^{p} \mathbf{F h}+\mathbf{w}^{p}, \mathbf{F}_{m, l}=e^{\frac{j 2 \pi k_{m} l}{K}},
$$

where $\mathbf{S}^{p}=\operatorname{diag}\left[d_{k_{1}}, d_{k_{2}}, \ldots, d_{k_{P}}\right]$. The least squares estimate of the channel impulse response corresponding to a candidate value of the time scale is now obtained as $\hat{\mathbf{h}}_{\tilde{a}}=\mathbf{F}^{\dagger}\left(\mathbf{S}^{p}\right)^{-1} \mathbf{x}_{\tilde{a}}^{p}$. The desired time scale parameter $a^{*}$ can then be finally determined using the maximum likelihood estimator [13] by searching over all candidate values and choosing the one which minimizes the metric:

$$
\begin{aligned}
a^{*} & =\arg \min _{\tilde{a}}\left|\mathbf{x}_{\tilde{a}}^{p}-\mathbf{S}^{p} \mathbf{F} \hat{\mathbf{h}}_{\tilde{a}}\right|^{2} \\
& =\arg \min _{\tilde{a}}\left|\mathbf{x}_{\tilde{a}}^{p}-\mathbf{S}^{p} \mathbf{F F} \mathbf{F}^{\dagger}\left(\mathbf{S}^{p}\right)^{-1} \mathbf{x}_{\tilde{a}}^{p}\right|^{2} .
\end{aligned}
$$

This minimization problem can be solved efficiently as it involves projecting a vector over a pre-determined subspace and then using a simple line search for evaluating $a^{*}$. Using the estimated time scale parameter, the channel impulse response is given as $\check{\mathbf{h}}=\mathbf{F}^{\dagger}\left(\mathbf{S}^{p}\right)^{-1} \mathbf{x}_{a^{*}}^{p}$. The transmitted symbols are now estimated by first computing the channel frequency response across all the subcarriers and then performing one-tap frequency domain equalization:

$$
\check{d}_{k}=\operatorname{dec}\left(\frac{x_{k}}{\check{H}_{k}}\right), \check{H}_{k}=\sum_{l=1}^{L} \check{h}_{l} e^{\frac{-j 2 \pi k l}{K}} .
$$

We conclude this section by emphasizing that the MW estimator is a good choice for scenarios in which a parametric representation of the combiner weights can be derived and the model parameters easily estimated.

\section{Performance Analysis: Theory}

In this section, we present an approximate theoretical analysis to characterize the performance of the proposed RW and MW estimators. The objective of this analysis is to provide intuition for choosing a practical value of $M$. We consider a CFO distorted OFDM system to highlight several key properties of partial FFT combining and the proposed estimators.

\section{A. Analysis of Model-Based Weight Estimation}

For the scenario of OFDM with CFO, we begin by assuming that the parameters of the optimal combiner are estimated correctly. Correcting for the phase distortion at the outputs of the partial FFT as in (25), we get $x_{k}=\sum_{m=1}^{M} e^{-j 2 \pi \epsilon \frac{2 m-1}{2 M}} y_{k}(m)$. Substituting for $y_{k}(m)$ from (5), the signal power at the output of the $k^{t h}$ subcarrier, $\mathrm{S}_{k}(M)$, can be evaluated as

$$
\mathrm{S}_{k}(M)=\left|H_{k}\right|^{2} \operatorname{sinc}^{2}\left(\frac{\pi \epsilon}{M}\right) .
$$

Similarly, the total noise-plus-interference power at the $k^{\text {th }}$ subcarrier, $N_{k}(M)$, is

$$
\begin{aligned}
\mathrm{N}_{k}(M)=N_{0}+\sum_{l=1, l \neq k}^{K}\left|H_{l}\right|^{2} \operatorname{sinc}^{2}\left(\frac{\pi(l-k+\epsilon)}{M}\right) \\
\times\left|\sum_{m=1}^{M} \frac{1}{M} e^{j 2 \pi(l-k) \frac{(2 m-1)}{2 M}}\right|^{2} .
\end{aligned}
$$

We observe that the interference term $\left|\sum_{m=1}^{M} \frac{1}{M} e^{j 2 \pi(l-k) \frac{(2 m-1)}{2 M}}\right|^{2}$ is non-zero only for those subcarriers for which $(l-k)$ is a multiple of $M$. Thus, for an OFDM system with frequency offset on a time-invariant channel, the partial FFT technique completely eliminates interference from all the subcarriers that are not at multiples of $M$ away from the subcarrier of interest. This is in contrast to a banded equalizer which only eliminates interference from only $2 M$ adjacent subcarriers, and thus significantly reduces detection error probability. The SIR of the $k^{t h}$ subcarrier for a fixed value of $M$ is given as

$$
\begin{aligned}
\operatorname{SIR}_{k}^{*}(M) & =\frac{S_{k}(M)}{N_{k}(M)} \\
& =\frac{\left|H_{k}\right|^{2} \operatorname{sinc}^{2}\left(\frac{\pi \epsilon}{M}\right)}{N_{0}+\sum_{l=1, l \neq k, l-k=p M}^{K}\left|H_{l}\right|^{2} \operatorname{sinc}^{2}\left(\frac{\pi(p M+\epsilon)}{M}\right)} .
\end{aligned}
$$

For a given channel frequency response, assuming that $\frac{\pi \epsilon}{M} \approx$ $\frac{\pi \epsilon}{M+1}, S I R_{k}^{*}(M)$ is a monotonically increasing function of $M$. Numerically it is always observed that $\operatorname{SIR}_{k}^{*}(M)$ monotonically increases with $M$ and thus choosing the largest value of $M$ gives the lowest symbol error probability. The optimal $M$ is then only limited by the computational available computational resources.

For OFDM signaling over time scale distorted UWA channels, even though the frequency offset is slightly different for each subcarrier, it can be treated as a constant in the neighborhood of each subcarrier. This suggests that significant improvement can be obtained for data detection over UWA channels in comparison to banded MMSE equalization.

\section{B. Analysis of Recursive Weight Estimation}

The RW estimation algorithm operates sequentially over subcarriers and its performance is a function of the rate of channel variation and the parameter $M$. Intuitively, as $M$ increases from a very small value ( $M=1$ is the minimum), the performance of the RW estimation improves as the distortion process is better modeled with increasing $M$. However, with a further increase in $M$, the number of weights to be estimated increases for a fixed amount of information available at the receiver, thus reducing the accuracy of estimating the combiner weights. Hence, we expect a performance degradation for larger values of $M$. To capture the tradeoff between modeling accuracy and overparametrization, we present a convergence analysis of the RLS under limiting conditions. As the number of subcarriers is finite and the channel is frequency selective, the data covariance matrix varies as a function of the subcarrier index, making a general analysis highly intractable. We consider several simplifying assumptions to facilitate our analysis.

As typical RLS convergence analysis assumes that the data covariance matrix $\left(E\left[\mathbf{y}_{k} \mathbf{y}_{k}^{H}\right]\right.$ in our case) is fixed [26], we make assumptions to approximate this as closely as possible. We begin by assuming that the channel is frequency flat with gain 
$h_{0}$. At the receiver, the signal experiences a frequency offset $f_{d}$ in addition to the AWGN at the input. The partial FFT outputs for the received signal can then be simplified from (9) as

$$
\begin{aligned}
\mathbf{y}_{k} & =\mathbf{D}_{\epsilon} \sum_{l=1}^{K} d_{l} h_{0} \mathbf{v}_{l-k}+\mathbf{n}_{k} \\
& =\mathbf{D}_{\epsilon} \sum_{l=1-k}^{K-k} d_{k+l} h_{0} \mathbf{v}_{l}+\mathbf{n}_{k}, \epsilon=f_{d} T .
\end{aligned}
$$

The data covariance matrix can now be expressed as

$$
\mathbf{R}_{\mathbf{y}_{k}}=\left|h_{0}\right|^{2} \mathbf{D}_{\epsilon}\left[\sum_{l=1-k}^{K-k} \mathbf{v}_{l} \mathbf{v}_{l}^{H}\right] \mathbf{D}_{\epsilon}^{H}+\frac{N_{0}}{M} \mathbf{I}_{M} .
$$

Substituting for $\mathbf{v}_{l}$ in the expression, we notice that the terms around $l=0$ contribute significantly to the covariance matrix while the magnitude of the contribution decreases as we move away from the subcarrier of interest. Using this observation allows us to treat the covariance matrix as being independent of $k$ for most of the subcarriers in the OFDM symbol. Deviations from this assumption are significant at the edge subcarriers, but are ignored for our analysis. Separating the signal and interference terms, we obtain

$$
\mathbf{R}=\left|h_{0}\right|^{2} \mathbf{D}_{\epsilon} \mathbf{v}_{0} \mathbf{v}_{0}^{H} \mathbf{D}_{\epsilon}^{H}+\mathbf{R}_{I},
$$

where $\mathbf{R}_{I}$ is the covariance matrix of the interfering terms and is given as

$$
\mathbf{R}_{I}=\left|h_{0}\right|^{2} \mathbf{D}_{\epsilon}\left[\sum_{l=1, l \neq k}^{K} \mathbf{v}_{l-k} \mathbf{v}_{l-k}^{H}\right] \mathbf{D}_{\epsilon}^{H}+\frac{N_{0}}{M} \mathbf{I}_{M} .
$$

Using the matrix inversion lemma, ${ }^{4}$ it can be shown that the data and interference covariance matrix are related as

$$
\frac{1}{\mathbf{v}_{0}^{H} \mathbf{R}^{-1} \mathbf{v}_{0}}=\left|h_{0}\right|^{2}+\frac{1}{\mathbf{v}_{0}^{H} \mathbf{R}_{I}^{-1} \mathbf{v}_{0}} .
$$

For the RW estimation algorithm using exponential decaying RLS with parameter $\lambda$, the optimization function for computing the combiner weights minimizes $\sum_{i=1}^{n} \lambda^{n-i}\left|h_{0} d_{i}-\mathbf{w}^{H}(n) \mathbf{y}_{i}\right|^{2}$.

For this scenario, the RLS adaptation rule is given by

$$
\begin{aligned}
e_{k} & =h_{0} d_{k}-\mathbf{w}_{k-1}^{H} \mathbf{y}_{k}, \\
\mathbf{w}_{k} & =\mathbf{w}_{k-1}+e_{k} \mathbf{g}_{k},
\end{aligned}
$$

where $e_{k}$ is the RLS prediction error at subcarrier $k$, and $g_{k}$ is the gain vector from the RLS update. Using the results from RLS convergence theory [27], we know that in the limit of large $k$, the mean weight vector $\mathbf{w}$ converges to the optimal linear MMSE solution: $\mathbf{w}_{*}=\left|h_{0}\right|^{2} \mathbf{R}^{-1} \mathbf{D}_{\epsilon} \mathbf{v}_{0}$. The SIR at the output of the optimal receiver can be computed as in [28]:

$$
\operatorname{SIR}^{*}(M)=\frac{E^{2}\left\{\mathbf{w}_{*}^{H} \mathbf{y}\right\}}{\operatorname{var}\left\{\mathbf{w}_{*}^{H} \mathbf{y}\right\}}=\left|h_{0}\right|^{2} \mathbf{v}_{0}^{H} \mathbf{D}_{\epsilon}^{H} \mathbf{R}^{-1} \mathbf{D}_{\epsilon} \mathbf{v}_{0} .
$$

We note that $\operatorname{SIR}^{*}(M)$ is derived by using the mid-point approximation for the received signal (see (6) in Section II), while the SIR evaluated in (35) uses the actual SIR by precisely evaluating all the integrals in (5).

To characterize the performance of the RLS algorithm, we compute the steady-state output error and the steady state SIR

\footnotetext{
${ }^{4}$ The matrix inversion identity for appropriately sized matrices A,B,C,D is given as $(A+B C D)^{-1}=A^{-1}-A^{-1} B\left(C^{-1}+D A^{-1} B\right)^{-1} D A^{-1}$.
}

at the output of the RLS [28]. The steady state error is the sum of two terms: the optimal MMSE error and the steady-state excess error[27], [28]. In the limit as the number of iterations tends to infinity, the steady state error at the output of the RLS in (40) converges to $\left|e_{k}\right|^{2} \rightarrow e_{*}+e_{e x}(\infty)$, where $e_{*}$ is the mean-square error obtained by optimal MMSE filtering using $\mathbf{w}_{*}$ [28]:

$$
\begin{aligned}
e_{*} & =E\left\{\left|h_{0} d_{k}-\mathbf{w}_{*}^{H} \mathbf{y}_{k}\right|^{2}\right\} \\
& =\left|h_{0}\right|^{2}\left(1-\left|h_{0}\right|^{2} \mathbf{v}_{0}^{H} \mathbf{D}_{\epsilon} \mathbf{R}^{-1} \mathbf{D}_{\epsilon} \mathbf{v}_{0}\right) \\
& =\frac{\left|h_{0}\right|^{2}}{1+\left|h_{0}\right|^{2} \mathbf{v}_{0}^{H} \mathbf{D}_{\epsilon} \mathbf{R}_{I}^{-1} \mathbf{D}_{\epsilon} \mathbf{v}_{0}}=\frac{\left|h_{0}\right|^{2}}{1+\mathrm{SIR}^{*}},
\end{aligned}
$$

and the steady-state excess mean-square error is given by $e_{e x}(\infty)=\frac{1-\lambda}{1+\lambda} M e_{*}=\eta e_{*}$ [28]. Now, under the assumption of known data symbols, the steady-state output SIR is defined as

$$
S I R^{\infty}=\lim _{k \rightarrow \infty} \frac{\left|E\left\{\mathbf{w}_{k-1}^{H} \mathbf{y}_{k}\right\}\right|^{2}}{\operatorname{var}\left\{\mathbf{w}_{k-1}^{H} \mathbf{y}_{k}\right\}} .
$$

The mean output value is

$$
\begin{aligned}
E\left\{\mathbf{w}_{k-1}^{H} \mathbf{y}_{k}\right\} & =\mathrm{E}\left\{\mathbf{w}_{k-1}^{H}\right\} \mathrm{E}\left\{\mathbf{y}_{k}\right\} \rightarrow d_{k} h_{0} \mathbf{w}_{*}^{H} \mathbf{D}_{\epsilon}^{H} \mathbf{v}_{0} \\
& =h_{0} d_{k} \frac{S I R^{*}}{1+S I R^{*}},
\end{aligned}
$$

where the first equality follows from the independence of $\mathbf{w}_{k-1}^{H}$ and $\mathbf{y}_{k}$. To compute the steady state output variance we first compute the second moment.

$$
\begin{aligned}
E\left\{\left|e_{k}\right|^{2}\right\}= & E\left\{\left|d_{k} h_{0}-\mathbf{w}_{k-1}^{H} \mathbf{y}_{k}\right|\right\} \\
= & \left|h_{0}\right|^{2}+E\left\{\left|\mathbf{w}_{k-1}^{H} \mathbf{y}_{k}\right|^{2}\right\} \\
& -2 h_{0} d_{k} E\left\{\mathbf{w}_{k-1}^{H} \mathbf{y}_{k}\right\} \\
\therefore E\left\{\left|\mathbf{w}_{k-1}^{H} \mathbf{y}_{k}\right|^{2}\right\}= & E\left\{\left|e_{k}\right|^{2}\right\}+2 h_{0} d_{k} E \\
& \times\left\{\mathbf{w}_{k-1}^{H} \mathbf{y}_{k}\right\} \\
& -\left|h_{0}\right|^{2} \rightarrow e_{*}+e_{e x}(\infty)-\left|h_{0}\right|^{2} \\
& \times\left(1-2 \mathbf{w}_{*}^{H} \mathbf{v}_{0}\right) .
\end{aligned}
$$

Using the mean and the second moment of the steady state output, the variance can be obtained as

$$
\operatorname{var}\left\{\mathbf{w}_{k-1}^{H} \mathbf{y}_{k}\right\} \rightarrow \frac{(1+\eta) S I R^{*}+\eta}{\left(1+S I R^{*}\right)^{2}} .
$$

The steady-state output SIR is then

$$
\begin{aligned}
S I R^{\infty} & =\lim _{n \rightarrow \infty} \frac{\left|E\left\{\mathbf{w}_{k-1}^{H} \mathbf{y}_{k}\right\}\right|^{2}}{\operatorname{var}\left\{\mathbf{w}_{k-1}^{H} \mathbf{y}_{k}\right\}} \\
& =\frac{S I R^{*}}{(1+\eta)+\frac{\eta}{\left(S I R^{*}\right)}},
\end{aligned}
$$

where $\eta=\frac{1-\lambda}{1+\lambda} M$ and $S I R^{*}(M)$ is a function of $M$ as given by (35). We observe that both the numerator and denominator of $S I R^{\infty}$ increase as $M$ increases. For smaller $M$, the parameter is very small and hence $S I R^{\infty}$ increases rapidly with $M$. For larger $M$, the numerator saturates and the denominator increases and hence $S I R^{\infty}$ reduces with increasing $M$. Hence there exists an optimal finite value of $M$ for which the RW estimator gives the best detection performance (see Section VI to see the optimal $M$ as a function of distortion parameters). 
TABLE I

COMPleXity Analysis With Perfect Channel State Information

\begin{tabular}{|c|c|}
\hline Algorithm & Order of Complex Multiplications \\
\hline Partial FFT general scenario & $O(M K \log K)+O\left(K M^{3}\right)+O\left(K M^{2} S\right)$ \\
\hline Partial FFT UWA scenario & $O(M K \log K)+O(K M)$ \\
\hline Serial MMSE [10] & $O(K \log K)+O\left(K D^{3}\right)$ \\
\hline MCMC based MAP-SDSC [15] & $O(K \log K)+O\left(K N_{d} D^{2}\right)$ \\
\hline PSE [14] & $O(K \log (K / M))+O(K D)$ \\
\hline
\end{tabular}

\section{Complexity Requirements}

The computational complexity of partial FFT combining is primarily determined by the algorithm used to compute the combiner weights, the number of subcarriers $K$ and the number of intervals $M$. To compute the partial FFT outputs, every algorithm begins by performing $M K$-point FFTs on a windowed version of the received signal thus having a maximum complexity of $M K \log K$ complex multiplications (CMs) (The actual complexity is much lesser than $M K \log K$ as $(M-1) \frac{K}{M}$ inputs to each FFT block are zeros). When perfect channel knowledge is assumed at the receiver, the optimal combiner weights are determined using (13) which involves solving a system of equations or computing the inverse of a matrix and approximately needs $\sim O\left(M^{3}\right)$ CMs. Computing the data covariance matrix in (13) using (14) requires the evaluation of a series with $K$ terms. The major contribution to $\mathbf{R}_{\mathbf{y}_{k}}$ is by the subcarriers in the neighborhood of the $k^{\text {th }}$ subcarrier and thus $\mathbf{R}_{\mathbf{y}_{k}}$ can be computed by a truncated version of the series in (14). The total complexity of computing the optimal combiner weights is then $O\left(M^{3}\right)+O\left(S M^{2}\right)$, where $S$ is the number of terms used in computing $\mathbf{R}_{\mathbf{y}_{k}}$.

For the special case of OFDM with time scale distortion or OFDM with CFO, the combiner weights on each subcarrier can be computed quickly using (28) or (25) respectively with a complexity of $M$ CMs and an additional $M$ CMs to perform the combining. Thus, by exploiting the special structure of the data covariance matrices, the partial FFT method enables computation of combiner weights and equalization in $O(M)$ $C M s$. A complete summary of the computational complexity of partial FFT combining and a comparison of the computational complexity of several other algorithms proposed in literature is summarized in Table I. The parameter $D$ in Table I is the number of sub/super diagonals considered for designing the banded equalizer and $N_{d}$ is the total number of iterations required to implement Gibbs sampling in [15]. Clearly for a general scenario such as DVB, the complexity of the partial FFT scheme is larger when compared to similar schemes in literature, but for the UWA scenario in which partial FFT combining exploits the structure of the time-varying channels, we clearly observe from Table I that the complexity of receiver processing is very small and scales linearly with $M$. In contrast, the MCMC based MAP-SDSC in [15] scales as $O\left(N_{d} D^{2}\right)$ while the serial MMSE scales as $O\left(D^{3}\right)$ for each subcarrier. Finally, though the PSE algorithm in [14] scales with $O(D)$, it is shown in [29] that the partial FFT technique significantly outperforms the PSE algorithm in [14].

The RW and MW estimators inherently combine channel estimation and computing combiner weights and hence the computational complexity of these estimators includes the cost of evaluating both sets of parameters. A summary of the total complexity of the RW and the MW estimator is given in Table II, assuming $P$ is the number of pilot symbols used, $L$ is the number of channel taps estimated and $N_{s}$ is the number of search points
TABLE II

COMPLEXITY ANALYSIS OF RW AND MW EstimatorS

\begin{tabular}{|c|c|}
\hline Algorithm & Number of Complex Multiplications \\
\hline RW estimator & $(M+1) K \log K+K\left(4 M^{2}+3 M+6\right)+P L$ \\
\hline MW estimator & $(M+1) K \log K+P N_{s}(M+2 L+1)$ \\
\hline
\end{tabular}

in the MW estimator. For the RW estimator, the computational complexity is dominated by the RLS algorithm which grows as $O\left(M^{2}\right)$. However, as shown in the analysis previously in this section, there exists an optimal value of $M$ for the RW estimator and increasing $M$ beyond this value does not improve the performance of the RW estimator. We will show in Section VI that the optimal value of $M$ is very small compared to $K$ (The maximum value of $M$ is $K$ ) and hence the RW estimator has moderate complexity. For the MW estimator, the complexity is dominated by the number of points used in the search for the optimal time-scale parameter $a^{*}(\operatorname{see}(31))$. Once the value of $a^{*}$ is determined the computation of the channel frequency response and then subsequently combiner weights can be computed using $O(M)$ operations.

\section{Performance Analysis: Simulation}

In this section, we evaluate the performance of the proposed partial FFT combining technique using numerical simulations. We primarily consider two scenarios (1) OFDM signaling over time-scale distorted UWA channel [2], [8], [9] and (2) DVB signaling over highly time-varying channels [5], [14], [15]. The simulation setup and the performance results for each of the scenarios is illustrated separately below.

\section{A. Underwater Acoustic Communications}

For UWA signaling, we consider an OFDM system with $K=$ 1024 subcarriers operating in a bandwidth $B=12 \mathrm{kHz}$ operating at a center frequency of $f_{c}=30 \mathrm{kHz}$. The subcarrier spacing is $\Delta f=12 \mathrm{~Hz}$ and the OFDM symbol duration is $T=\frac{1}{\Delta} f=83.3 \mathrm{~ms}$. A cyclic prefix of length $T_{g}=$ $\frac{T}{8}=10.41 \mathrm{~ms}$ is added to the OFDM symbol to eliminate inter-symbol interference from the previous symbol. The normalized relative velocity between the transmitter and the receiver is represented by $a=\frac{v}{c}$, where $v$ is the relative velocity between the communicating nodes and $c=1500 \frac{\mathrm{m}}{\mathrm{sec}}$ is the speed of sound in water. The value of $a$ can reach values in excess of $a=10^{-3}$ for mobile underwater nodes [8], [9], [13]. As a first step to removing the time-scale distortion, the received signal is resampled using a coarse estimate of $\frac{v}{c}$. However, even a small error in estimating $a$ results in residual time scaling on the order of $a=10^{-4}$. We assume that $a$ is a uniformly distributed random variable on the support $\left[-1.5 \times 10^{-4}, 1.5 \times\right.$ $\left.10^{-4}\right]$ for our simulations. As the time-scale distortion results in a different frequency shift for each subcarrier, the normalized Doppler for UWA OFDM is a function of the subcarrier frequency. For the system under consideration, the normalized Doppler can be computed as $a f_{k} T$ which is 0.2 for the first subcarrier to about 0.3 for the last subcarrier assuming $a=10^{-4}$. A normalized Doppler of about 0.2 causes strong ICI which spans over several tens of subcarriers. To simulate the underwater channel, we consider a 6-tap sparse channel with power profile $[0,-0.9,-4.9,-8.0,-7.8,-23.7] \mathrm{dB}$ and a delay profile with the first tap arriving at $0 \mathrm{~ms}$ and last tap arriving at $5 \mathrm{~ms}$. 


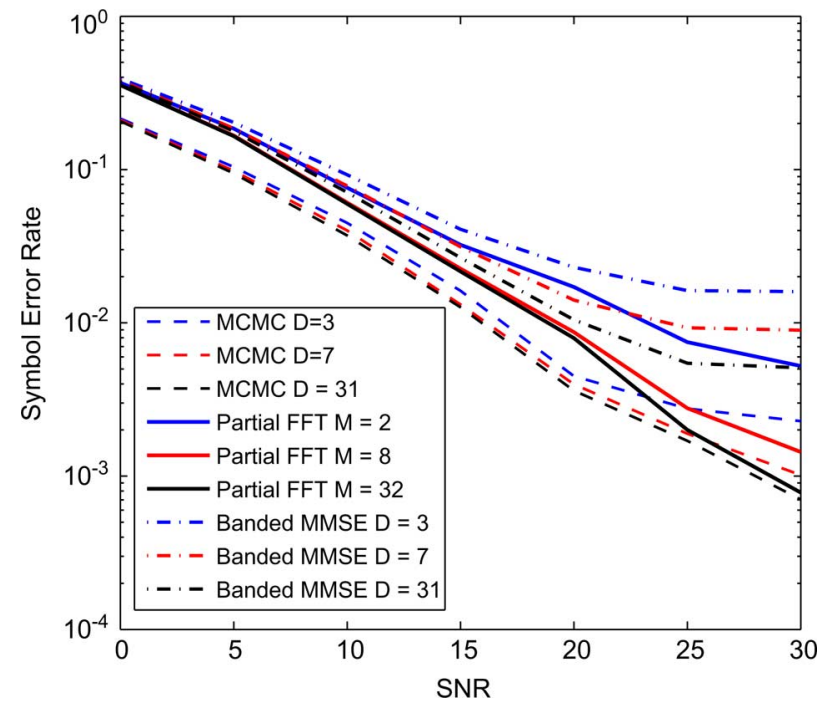

Fig. 1. Symbol Error Rate assuming perfect knowledge of the UWA channel as a function of SNR and model parameters for the partial FFT combiner, banded MMSE and MCMC based MAP SDSC [15].

Fig. 1 shows the Symbol Error Rate (SER) for the partial FFT combiner, banded MMSE equalizer and the MCMC based MAP sequence detection with successive cancelation (SDSC) scheme in [15] as a function of SNR and the model parameters. Assuming perfect knowledge of the time-varying channel, the partial FFT combiner weights have been computed using (28). The combiner weights can be computed in $O(M)$ time, however they are not optimal due to the circulant approximation of the Toeplitz-Hermitian matrix (see Section IV for a detailed discussion). For the MCMC based MAP-SDSC in [15], perfect channel state information is available, the interference from all the previous symbols is subtracted out and only the interference from the future symbols is accounted into the receiver design. Gibbs sampling with $N_{d}=30$ iterations is used to compute the MAP estimates of symbols with the first 10 iterations being considered as the burn-in period. The complexity of computing the combiner weights is $O\left(N_{d} D^{2}\right)$. From Fig. 1, it is observed that the partial FFT combiner performs significantly better than the standard banded MMSE equalizer whose complexity is $O\left(D^{3}\right)$ per symbol while approaching the performance of the MCMC based MAP SDSC in [15] at moderate to high SNR. Thus, we observe that by exploiting the structure of the UWA time-variation, partial FFT combining can eliminate significant amount of interference and attain a performance close to higher complexity and interference canceling data detectors. We also note that the partial FFT method is a pre-processing technique that can be used in combination with the MCMC based MAP SDSC and other post-FFT processing techniques as well.

Fig. 2 shows the SER obtained when performing data detection using the RW and MW estimator. The RW estimator does not assume prior knowledge of the time-varying channel while the MW estimator only assumes knowledge of the distortion process. The RW estimator uses the initialization parameters as shown in Algorithm 1 (see Section III). The parameters $\lambda$ and $\alpha$ which control the RLS has been numerically optimized to give the best SER. Pilots have been placed uniformly throughout the OFDM symbol with 7 data symbols between each pilot. We note that while some techniques such as [8], [9] use zero symbols surrounding the pilots to reduce ICI experienced by the pilots, our

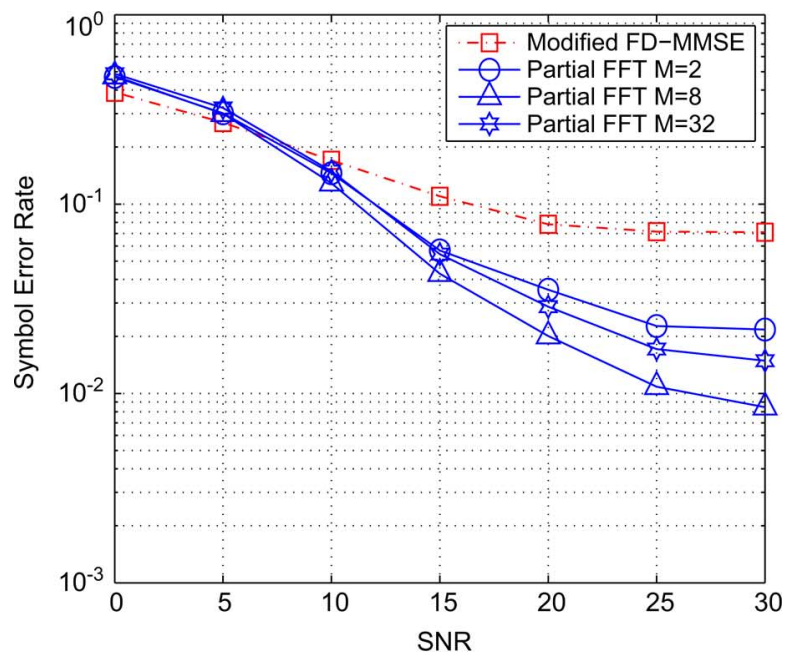

(a)

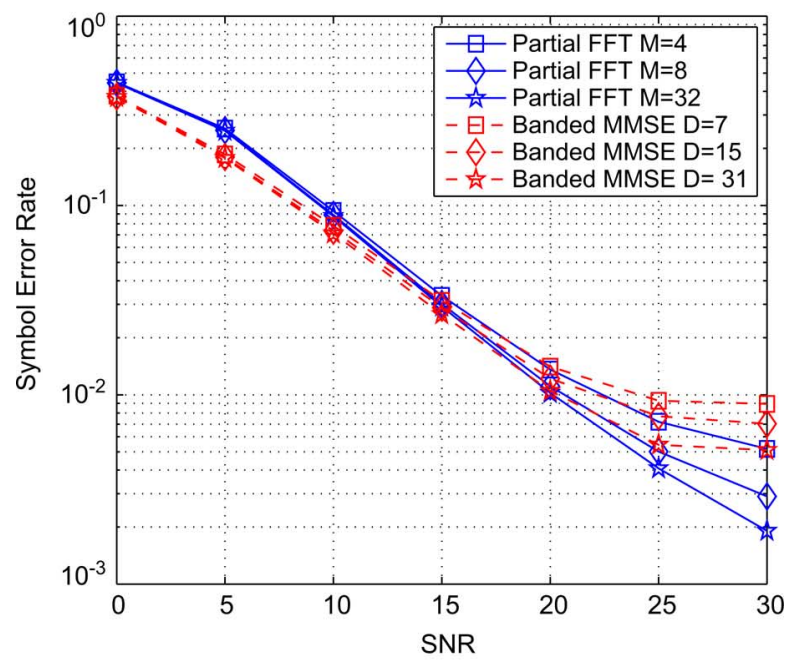

(b)

Fig. 2. Symbol Error Rate of the RW and MW estimators as a function of SNR and model parameters (a) RW estimator (b) MW estimator.

method does not place zero symbols around pilots. However, to train the adaptive equalizer, we assume that the first $2 M$ subcarriers of each OFDM symbol are pilots. For the RW estimator in Fig. 2(a), we observe that at moderate SNR, the SER improves with increasing $M$ as the time-varying channel is better compensated for thus resulting in reduced ICI. As $M$ increases further, the number of weights to be estimated increases causing over-parametrization and reduced accuracy of estimating the desired parameters. For example, we see from Fig. 2(a) that the performance is the best when $M=8$ and a degradation is observed for $M=2$ and $M=32$. Observe that this behavior has been predicted by the analysis of OFDM over flat fading channels with CFO in Section V (see (47)). As the RW estimator does not completely eliminate ICI, an error floor is observed at high SNR due to the residual ICI.

Fig. 2(a) also shows a comparison between the RW estimator and a modified version of [18]. Both methods use adaptive algorithms to estimate parameters relevant to ICI compensation and neither assumes any knowledge of the channel and both methods use the same number of pilots for a fair comparison. The channel estimates are obtained adaptively (LMS is used in [18]) using pilots and tentative decisions (single tap equalization 


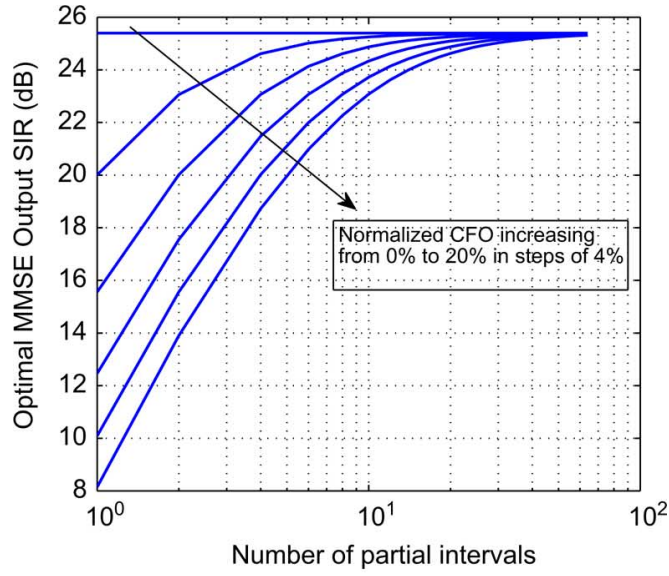

(a)

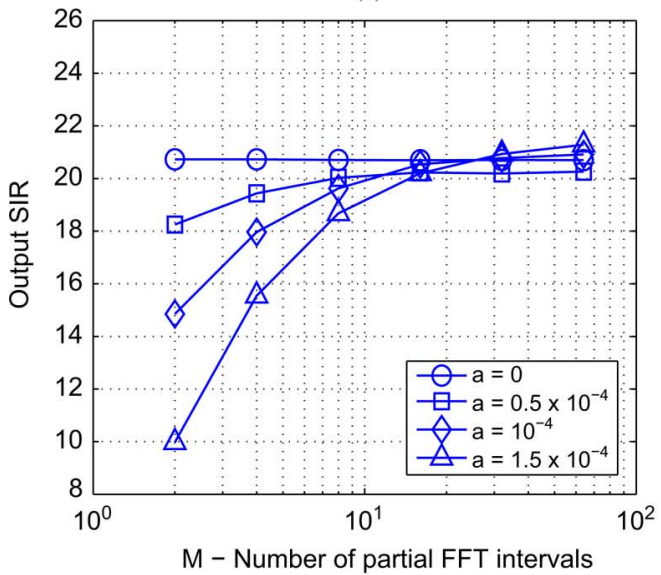

(b)

Fig. 3. Output SIR as a function of $M$ for an input SNR of $25 \mathrm{~dB}$ for a UWA OFDM system using MW estimation and partial FFT combining (a) Flat fading channel with CFO (b) Frequency selective channel with time scale distortion.

and detection ignoring ICI). The estimated ICI is then subtracted from the received data for each subcarrier, and equalization is performed to get new data symbol estimates. In our comparison, we select the best value of $D$ (one which gives lowest SER) based on numerical simulations. It is clearly observed that the RW method significantly outperforms the method in [18] under the current simulation scenario.

For the MW estimator in Fig. 2(b), we assume that $\frac{K}{8}=128$ pilots are placed uniformly throughout the OFDM symbol, the channel length $L$ conservatively set at $L=80$ taps and the number of search points in (31) for determining the optimal time-scale parameter chosen to be $N_{s}=60$. From Fig. 2(b), we see that for the MW estimator the SER improves significantly with increasing $M$. As $M$ grows larger, the performance improvement saturates and results in a tradeoff due to the increasing complexity. Fig. 2(b) also shows a comparison between the MW estimator (which estimates the channel and distortion parameters) and the banded MMSE equalizers (which assume perfect channel knowledge). We clearly observe that even when there is a difference in channel knowledge, the MW estimator significantly outperforms the conventional banded MMSE equalizer at moderate to high SNRs at a much lower complexity compared to the banded MMSE equalizer.

1) Comparison of Simulation and Analytical Results: The analysis in Section V assumes a frequency offset scenario,

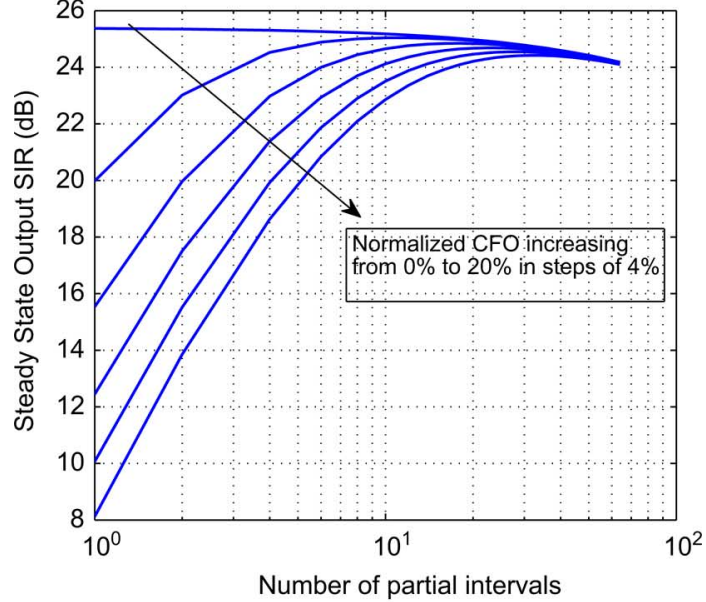

(a)

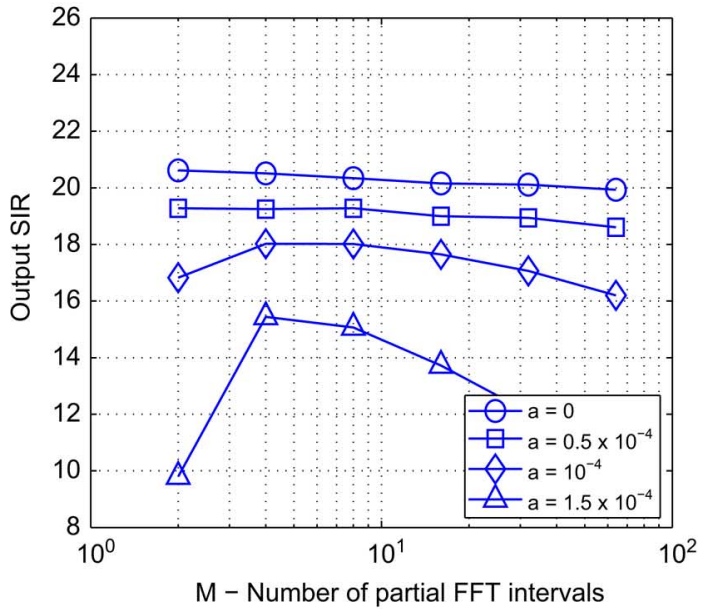

(b)

Fig. 4. Output SIR as a function of $M$ for an input SNR of $25 \mathrm{~dB}$ for a UWA OFDM system using RW estimation and partial FFT combining (a) Flat fading channel with CFO (b) Frequency selective channel with time scale distortion.

which is a simplification of the more general time scale distortion scenario. To assess the predictive value of these analytical results, we examine the two scenarios: OFDM with $\mathrm{CFO}$ and time-scale distortion.

Fig. 3(a) shows the optimal SIR as a function of the number of OFDM sub-intervals $M$ for various values of the normalized CFO and an input SNR of $25 \mathrm{~dB}$. The optimal SIR is the output SIR of the MW estimator which has perfect knowledge of the channel and the frequency offset. As pointed out in Section V, the optimal SIR is a monotonically increasing function of $M$, since the mid-point approximation becomes more accurate with increasing $M$. We observe that the theoretical output SIR reaches its steady state value even for small values of $M$. Fig. 3(b) shows the pre-detection SIR for the UWA channel with MW estimation for various values of $M$ and time-scale distortion. All the required channel and time-scale parameters are estimated during the course of the simulation. We observe that except for a small loss in the maximum output SIR (from $25 \mathrm{~dB}$ to around $21 \mathrm{~dB}$ ) due to errors in channel and time scale estimation, the plot of the output SIR is as predicted in Fig. 3(a) thus validating our approximate analysis.

Fig. 4(a) shows the theoretically expected SIR output for OFDM signaling over a flat fading channel with CFO for 


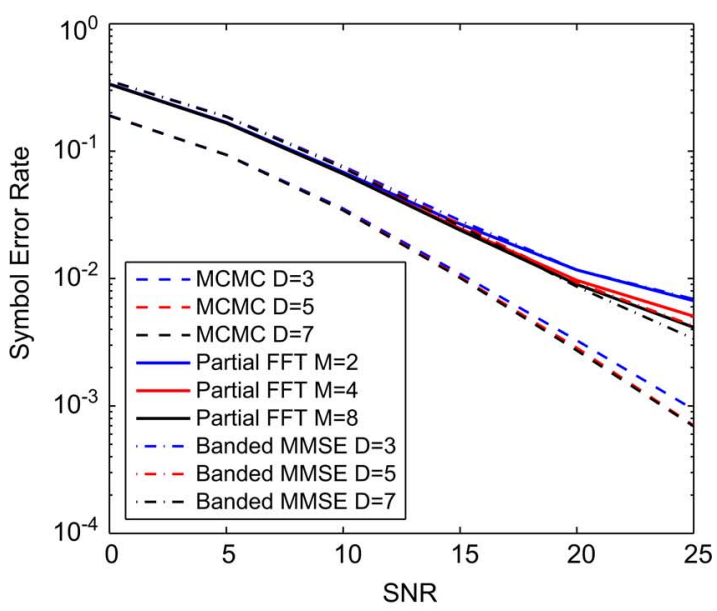

(a)

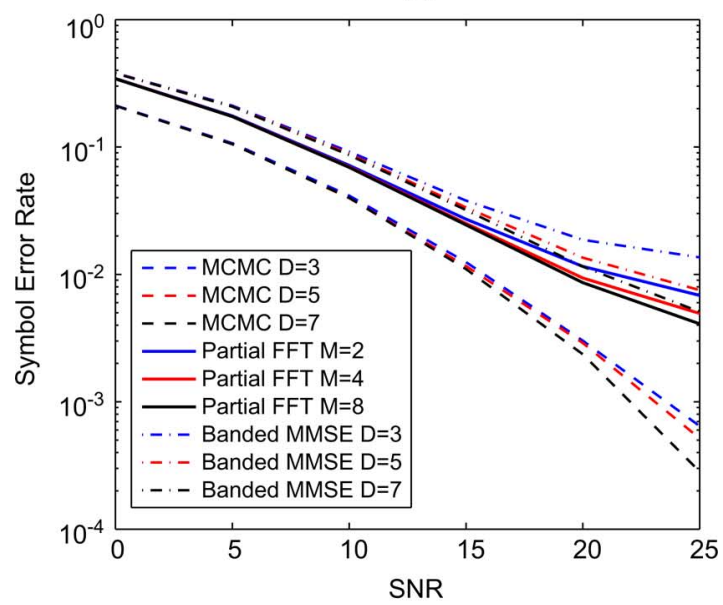

(b)

Fig. 5. SER assuming perfect knowledge of the DVB wireless channel as a function of SNR and model parameters. (a) Velocity $=180 \mathrm{kmph}$, Normalized Doppler $=0.11$. (b) Velocity $=240 \mathrm{kmph}$, Normalized Doppler $=0.15$.

various values of normalized $\mathrm{CFO}$ and $M$ when all the system and channel parameters are known perfectly. While the $S I R^{*}$ in (47) increases with $M$ due to more accurate modeling, it saturates at moderate values of $M$. However, the parameter $\eta$ that characterizes the adaptive algorithm, increases with $M$, slowly reducing the steady-state SIR. The SIR curve thus exhibits a maximum, which indicates the optimal number of partial intervals to be used. We also observe from Fig. 4(a) that the slope of the SIR curve is different on both sides of the optimal $M$, and hence, erring on the side of larger $M$ is better than choosing a smaller value. Fig. 3(b) show the pre-detection SIR for an UWA channel with RW estimation for frequency selective fading channel. We observe that the trend of the SIR curve is as predicted by the theoretical analysis. However, as the curve in Fig. 4(a) is for a simplified OFDM system with flat fading and CFO with known parameters, and the curve in Fig. 4(b) shows the measured output for a time scaled OFDM system with estimated parameters, there is a mismatch between the two figures which can be attributed to the time-varying nature of the data covariance matrix. However, the purpose of the analysis is to provide intuition for choosing an optimal value of $M$ and we note from Fig. 4(b) that choosing $M$ between 8 and 16 is optimal depending on the level of expected Doppler distortion and agrees well with the results in Fig. 2(a).

\section{B. Digital Video Broadcasting}

For DVB signaling between mobile devices, we consider an OFDM signal with $K=2048$ subcarriers in a bandwidth of $B=2 \mathrm{MHz}$ centered at $f_{c}=650 \mathrm{MHz}$. The subcarrier spacing is $\Delta f=976.5 \mathrm{~Hz}$ and the OFDM symbol duration is $T=1 \mathrm{~ms}$. The cyclic prefix is $T_{g}=\frac{T}{8}=125 \mu \mathrm{s}$. The wireless channel between the mobile antenna and the receiver are modeled based on a realistic channel model determined by the COST-207 project and the Typical Urban TU-6 channel model is considered for simulation [15]. The TU-6 channel model has 6 taps and the multipath gains on each tap are modeled with the Jakes model. We consider velocities of $180 \mathrm{kmph}$ and $240 \mathrm{kmph}$ which result in a normalized Doppler of $f_{d} T=0.11$ and 0.15 approximately.

Figs. 5(a) and 5(b) show the SER as a function of various system parameters for the partial FFT based combiner, banded MMSE equalizer and the MAP based SDSC algorithm in [15] as a function of SNR for velocities $180 \mathrm{kmph}$ and $240 \mathrm{kmph}$ respectively. We observe that in general the partial FFT method performs better than the banded MMSE equalizer of equivalent complexity but there exists a performance gap when compared to the MAP based SDSC. This can be attributed to the fact that the MAP based SDSC is an interference canceling equalizer and thus reduces the effective amount of interference seen by each data symbol.

\section{CONCLUSIONS}

In this paper, we considered the problem of OFDM data detection over a highly time-varying channel. A detection technique called partial FFT demodulation was proposed, wherein each OFDM symbol is divided into several smaller intervals and FFT is performed on each. Weighted combining of the partial FFT outputs results in a significant reduction in ICI compared to banded equalization and permits low complexity symbol-bysymbol detection. While banded equalization typically accounts for ICI only from a few adjacent subcarriers, partial FFT demodulation can take into account ICI from many more subcarriers without increasing the computational complexity. Three scenarios were considered in which either full, partial or no knowledge of the channel is available, and techniques for deriving the optimum combiner weights in each of these scenarios have been presented. An approximate theoretical analysis of the proposed weight estimators was presented, showing interesting properties of the estimators that explain the trends observed in numerical simulations. Numerical results for time-varying channels corresponding underwater acoustic communications and digital video broadcasting demonstrate the significant improvements that can be obtained using the proposed technique.

\section{ACKNOWLEDGMENT}

The authors would like to thank Dr. Zijian Tang for suggesting the comparison in Section II-E and the reviewers for several valuable comments in improving the quality of this paper.

\section{REFERENCES}

[1] S. Yerramalli, M. Stojanovic, and U. Mitra, "Partial FFT demodulation: A detection method for Doppler distorted OFDM systems," in Proc. Int. Workshop on Signal Process. Adv. Wireless Commun. (SPAWC), 2010, pp. 1-5.

[2] S. Yerramalli, M. Stojanovic, and U. Mitra, "Data detection techniques for OFDM signals over Doppler-distorted channels," in WUWNet '10: Proc. 4th ACM Int. Workshop on UnderWater Netw., 2010, pp. 1-4. 
[3] S. Yerramalli, M. Stojanovic, and U. Mitra, "Analysis of partial FFT demodulation for Doppler distorted OFDM signals," in Proc. IEEE Asilomar Conf. Signals, Syst. Comput., Nov. 2010, pp. 1060-1064.

[4] D. Astely, E. Dahlman, A. Furuskar, Y. Jading, M. Lindstrom, and S Parkvall, "LTE: The evolution of mobile broadband," IEEE Commun. Mag., pp. 44-51, Apr. 2009.

[5] DVB-CM-NGH, Commercial Requirements for DVB-NGH 2009.

[6] L. Vangelista, N. Benvenuto, S. Tomasin, C. Nokes, J. Stott, A. Filippi, M. Vlot, V. Mignone, and A. Morello, "Key technologies for next-generation terrestrial digital television standard DVB-T2," IEEE Commun. Mag., vol. 47, no. 10, pp. 146-153, Oct. 2009.

[7] M. Stojanovic, "MIMO OFDM over underwater acoustic channels," in Proc. 43rd Asilomar Conf. Signals, Syst. Comput., Nov. 2009, pp. 605-609.

[8] B. Li, S. Zhou, M. Stojanovic, L. Freitag, and P. Willet, "Multicarrier communication over underwater acoustic channels with nonuniform Doppler shifts," IEEE J. Ocean. Eng., vol. 33, no. 2, pp. 198-209, Apr. 2008.

[9] J. Huang, S. Zhou, J. Huang, C. R. Berger, and P. Willett, "Progressive inter-carrier interference equalization for OFDM transmission over time-varying underwater acoustic channels," IEEE J. Sel. Topics in Signal Process., vol. 5, no. 8, pp. 1524-1536, 2011.

[10] P. Schniter, "Low-complexity equalization of OFDM in doubly selective channels," IEEE Trans. Signal Process., vol. 52, no. 4, pp. 1002-1011, Apr. 2004

[11] K. Fang, L. Rugini, and G. Leus, "Low-complexity block turbo equalization fo OFDM systems in time-varying channels," IEEE Trans. Signal Process., vol. 56, no. 11, pp. 5555-5566, Nov. 2008.

[12] L. Rugini, P. Banelli, and G. Leus, "Low complexity banded equalizers for OFDM systems in Doppler spread channels," Eurasip J. Appl. Signal Process., no. 67404, pp. 1-13, 2006.

[13] A. B. Salberg and A. Swami, "Doppler and frequency offset synchronization in wideband OFDM," IEEE Trans. Wireless Commun., vol. 4 no. 6, pp. 2870-2881, Nov. 2006.

[14] P. Baracca, S. Tomasin, L. Vangelista, N. Benvenuto, and A. Morello, "Per sub-block equalization of very long OFDM blocks in mobile communications," IEEE Trans. Commun., vol. 59, no. 2, pp. 363-368, Feb. 2011.

[15] E. Panayirci, H. Dogan, and H. Poor, "Low-complexity MAP-based successive data detection for coded OFDM systems over highly mobile wireless channels," IEEE Trans. Veh. Technol., vol. 60, pp. 2849-2857, Jul. 2011.

[16] P. C. Carrascosa and M. Stojanovic, "Adaptive channel estimation and data detection for underwater acoustic MIMO-OFDM systems," IEEE J. Ocean. Eng., vol. 35, no. 3, pp. 635-646, Jul. 2010.

[17] S. Sen and A. Nehorai, "Adaptive design of OFDM radar signal with improved wideband ambiguity function," IEEE Trans. Signal Process., vol. 58, no. 2, pp. 928-933, Feb. 2010.

[18] K. Tu, D. Fertonani, T. M. Duman, M. Stojanovic, J. G. Proakis, and P. Hursky, "Mitigation of intercarrier interference for OFDM systems for time-varying underwater acoustic channels," IEEE J. Ocean. Eng., vol. 36, no. 2, pp. 156-171, Apr. 2011.

[19] M. Stojanovic, "An adaptive algorithm for differentially coherent detection in the presence of intersymbol interference," IEEE J. Sel. Areas Commun., vol. 23, no. 9, pp. 1884-1890, Sep. 2005.

[20] H. Zhang, X. Dai, and D. Li, "Low-complexity equalization of rapidly time-varying multi-path channels for OFDM systems," in Proc. IEEE Int. Conf. Inf., Commun., Signal Process., Dec. 2009, pp. 1-4.

[21] M. Martone, "A multicarrier system based on the fractional Fourier transform for time-frequency-selective channels," IEEE Trans. Commun., vol. 49, no. 6, pp. 1011-1020, Jun. 2001.

[22] T. Erseghe, N. Laurenti, and V. Cellini, "A multicarrier architecture based upon the affine Fourier transform," IEEE Trans. Commun., vol. 53, no. 5, pp. 853-862, May 2005.

[23] K. Liu, T. Kadous, and A. M. Sayeed, "Orthogonal time-frequency signaling over doubly dispersive channels," IEEE Trans. Inf. Theory, vol. 50, no. 11 , pp. 2583-2603, Nov. 2004

[24] J. Li, G. Liu, and G. B. Giannakis, "Carrier frequency offset estimation for OFDM-based WLANs," IEEE Signal Process. Lett., vol. 8, no. 3 , pp. 80-82, Mar. 2001.

[25] R. M. Gray, Toeplitz and Circulant Matrices: A Review.. New York: Now.

[26] W. Chen and U. Mitra, "An improved blind adaptive MMSE receiver for fast fading DS-CDMA channels," IEEE J. Sel. Areas Commun., vol. 19, no. 8, pp. 1531-1543, Aug. 2001.

[27] E. Elefteriou and D. D. Falconer, "Tracking properties and steady-state performance of RLS adaptive filter algorithms," IEEE Trans. Acoust., Speech Signal Process., vol. 34, no. 5, pp. 1097-1110, Oct. 1986.

[28] H. V. Poor and X. Wang, "Code-aided interference supression for DS/CDMA communications-part II: Parallel blind adaptive implementations," IEEE Trans. Commun., vol. 45, no. 9, pp. 1112-1122, Sep. 1997.
[29] P. Baracca, S. Tomasin, and N. Benvenuto, "Equalization of OFDM for doubly very selective channels," in Proc. IEEE Int. Conf. Commun. Technol., Nov. 2010, pp. 29-32.

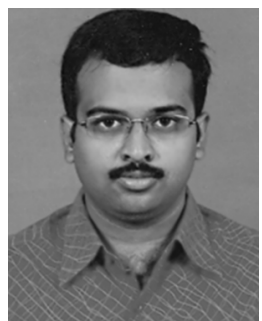

Srinivas Yerramalli (S'03) received the B.Tech. degree from the International Institute of Information Technology in 2007 and the M.S. degree from the University of Southern California, Los Angeles, in 2008.

$\mathrm{He}$ is a Ph.D. degree candidate in the Ming Hsieh Department of Electrical Engineering, University of Southern California. His research primarily focuses on physical layer communications for highly time-varying channels especially Underwater Acoustic Channels and in understanding the feasbility of cooperation in heterogenous wireless networks. More broadly, he is interested in problems related to signal processing and optimization for wireless communication systems. He interned at Qualcomm CDMA Technologies in the summer of 2010 and in the Mobility, Networks and Systems group at Microsoft Research in the summer of 2011.

Mr. Yerramalli is a recipient of the Ming Hsieh Scholar award for 2011-2012, the IIIT Gold Medal for excellent academic performance in 2007, the Prathibha Scholarship from the Government of Andhra Pradesh from 2003 to 2007, and a Gold Medal from the President of India for his performance in the All India Engineering Entrance Examination (AIEEE) in 2003.

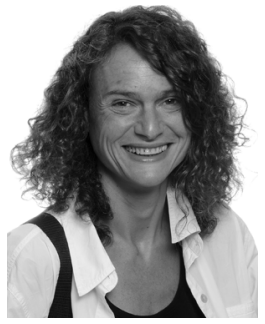

Milica Stojanovic (SM'08-F'10) graduated from the University of Belgrade, Serbia, in 1988, and received the M.S. and Ph.D. degrees in electrical engineering from Northeastern University, Boston, MA, in 1991 and 1993, respectively.

She was a Principal Scientist at the Massachusetts Institute of Technology (MIT), Cambridge, until 2008, when she joined Northeastern University, Boston, where she is currently a Professor of Electrical and Computer Engineering. She is also a Guest Investigator at the Woods Hole Oceanographic Institution, and a Visiting Scientist at MIT. Her research interests include digital communications theory, statistical signal processing and wireless networks, and their applications to underwater acoustic systems.

Dr. Milica is an Associate Editor for the IEEE JOURNAL OF OCEANIC ENGINEERING and the IEEE TRANSACTIONS ON SIGNAL PROCESSING.

Urbashi Mitra (F'07) received the B.S. and the M.S. degrees from the University of California at Berkeley and the Ph.D. degree from Princeton University, Princeton, NJ.

After six years with the Ohio State University, she joined the Department of Electrical Engineering, University of Southern California, Los Angeles, where she is currently a Professor. She has held visiting appointments at the Delft University of Technology, The Netherlands; Stanford University, Stanford, CA; Rice University, Houston, TX; and the Eurecom Institute.

Dr. Mitra has been an Associate Editor for the following IEEE publications: TRANSACTIONS ON INFORMATION THEORY (2007-2011), JOURNAL OF OCEANIC ENGINEERING (2006-2011), and TRANSACTIONS ON COMMUNICATIONS (19962001). She was a member of the IEEE Information Theory Society's Board of Governors (2002-2007) and began a third term in 2012. She is also a member of the IEEE Signal Processing Society's Technical Committee on Signal Processing for Communications and Networking (2012-2014). She is the recipient of: 2012 NAE Lillian Gilbreth Lectureship, 2011 USC Zumberge Interdisciplinary Innovation Fund (with M. El-Naggar), USC Center for Excellence in Research Fellowship (2010-2013), the Viterbi School of Engineering Dean's Faculty Service Award (2009), USC Mellon Mentoring Award (2008), Texas Instruments Visiting Professor (Fall 2002, Rice University), 2001 Okawa Foundation Award, 2000 Lumley Award for Research (OSU College of Engineering), 1997 MacQuigg Award for Teaching (OSU College of Engineering), and a $1996 \mathrm{Na}-$ tional Science Foundation (NSF) CAREER Award. She served as co-Director of the Communication Sciences Institute, University of Southern California, from 2004 to 2007. 